\title{
Improvements of bounds for the Sándor-Yang means
}

Wei-Mao Qian' ${ }^{1}$ Hui-Zuo Xu and Yu-Ming Chu ${ }^{3 *}$ (D)

"Correspondence:

chuyuming2005@126.com

${ }^{3}$ Department of Mathematics, Huzhou University, Huzhou, China

Full list of author information is available at the end of the article

\begin{abstract}
In the article, we provide new bounds for two Sándor-Yang means in terms of the arithmetic and contraharmonic means. Our results are the improvements of the previously known results.
\end{abstract}

MSC: $26 \mathrm{E} 60$

Keywords: Sándor-Yang mean; Arithmetic mean; Quadratic mean; Contra-harmonic mean; Neuman-Sándor mean; Seiffert mean; Power mean; Schwab-Borchardt mean

\section{Introduction}

Let $p \in \mathbb{R}$ and $x, y>0$ with $x \neq y$. Then the arithmetic mean $A(x, y)$, quadratic mean $Q(x, y)$, contraharmonic mean $C(x, y)$, Neuman-Sándor mean $N S(x, y)$ [1], Seiffert mean $T(x, y)$ [2-5], $p$ th power mean $M_{p}(x, y)$ [6-13], and Schwab-Borchardt mean $S B(x, y)[14,15]$ are defined by

$$
\begin{aligned}
& A(x, y)=\frac{x+y}{2}, \quad Q(x, y)=\sqrt{\frac{x^{2}+y^{2}}{2}}, \quad C(x, y)=\frac{x^{2}+y^{2}}{x+y}, \\
& N S(x, y)=\frac{x-y}{2 \sinh ^{-1}\left(\frac{x-y}{x+y}\right)}, \quad T(x, y)=\frac{x-y}{2 \arctan \left(\frac{x-y}{x+y}\right)}, \\
& M_{p}(x, y)= \begin{cases}\left(\frac{x^{p}+y^{p}}{2}\right)^{1 / p}, & p \neq 0, \\
\sqrt{x y}, & p=0,\end{cases}
\end{aligned}
$$

and

$$
S B(x, y)= \begin{cases}\frac{\sqrt{y^{2}-x^{2}}}{\arccos (x / y)}, & x<y, \\ \frac{\sqrt{x^{2}-y^{2}}}{\cosh ^{-1}(x / y)}, & x>y,\end{cases}
$$

respectively, where $\sinh ^{-1}(t)=\log \left(t+\sqrt{t^{2}+1}\right)$ and $\cosh ^{-1}(t)=\log \left(t+\sqrt{t^{2}-1}\right)$ are the inverse hyperbolic sine and cosine functions.

Let $U(x, y)$ and $V(x, y)$ be the symmetric bivariate means. Then Yang [16] introduced the Sándor-Yang mean

$$
R_{U V}(x, y)=: V(x, y) e^{\frac{U(x, y)}{S B[U(x, y), V(x, y)]}-1},
$$

(c) The Author(s) 2019. This article is distributed under the terms of the Creative Commons Attribution 4.0 International License (http://creativecommons.org/licenses/by/4.0/), which permits unrestricted use, distribution, and reproduction in any medium, provided you give appropriate credit to the original author(s) and the source, provide a link to the Creative Commons license, and indicate if changes were made. 
and provided the explicit formulas for $R_{A Q}(x, y)$ and $R_{Q A}(x, y)$ as follows:

$$
\begin{aligned}
& R_{A Q}(x, y)=Q(x, y) e^{A(x, y) / T(x, y)-1} \\
& R_{Q A}(x, y)=A(x, y) e^{Q(x, y) / N S(x, y)-1} .
\end{aligned}
$$

Recently, the bounds and properties for certain bivariate means and related special functions have attracted the attention of many researchers [17-28].

Zhao, Qian, and Song [29] proved that the double inequalities

$$
\begin{aligned}
& M_{\alpha}(a, b)<R_{Q A}(a, b)<M_{\beta}(a, b), \\
& M_{\lambda}(a, b)<R_{A Q}(a, b)<M_{\mu}(a, b)
\end{aligned}
$$

hold for all $a, b>0$ with $a \neq b$ if and only if $\alpha \leq \log 2 /[1+\log 2-\log (1+\sqrt{2})]=1.5517 \ldots$, $\beta \geq 5 / 3, \lambda \leq 4 \log 2 /(4+2 \log 2-\pi)=1.2351 \ldots$, and $\mu \geq 4 / 3$.

$\mathrm{Xu}$ [30], and $\mathrm{Xu}, \mathrm{Chu}$, and Qian [31] proved that the two-sided inequalities

$$
\begin{aligned}
& C^{1 / 6}(x, y) A^{5 / 6}(x, y)<R_{A Q}(x, y)<\frac{1}{6} C(x, y)+\frac{5}{6} A(x, y), \\
& C^{1 / 3}(x, y) A^{2 / 3}(x, y)<R_{Q A}(x, y)<\frac{1}{3} C(x, y)+\frac{2}{3} A(x, y)
\end{aligned}
$$

are valid for all $x, y>0$ with $x \neq y$.

The main purpose of this paper is to improve the bounds for $R_{A Q}(x, y)$ and $R_{Q A}(x, y)$ given by (1.5) and (1.6).

\section{Lemmas}

In order to prove our main results, we need four lemmas which we present in this section.

Lemma 2.1 (see [32, Theorem 1.25]) Let $a, b \in \mathbb{R}$ with $a<b, f, g:[a, b] \rightarrow \mathbb{R}$ be continuous on $[a, b]$ and differentiable on $(a, b)$, and $g^{\prime}(x) \neq 0$ on $(a, b)$. If $f^{\prime}(x) / g^{\prime}(x)$ is increasing (decreasing) on $(a, b)$, then so are the functions

$$
\frac{f(x)-f(a)}{g(x)-g(a)}, \quad \frac{f(x)-f(b)}{g(x)-g(b)} .
$$

If $f^{\prime}(x) / g^{\prime}(x)$ is strictly monotone, then the monotonicity in the conclusion is also strict.

Lemma 2.2 (see [33, Lemma 1.1]) Suppose that the power series $f(x)=\sum_{n=0}^{\infty} a_{n} x^{n}$ and $g(x)=\sum_{n=0}^{\infty} b_{n} x^{n}$ have the radius of convergence $r>0$, and $b_{n}>0$ for all $n=0,1,2, \ldots$ If there exists $n_{0} \geq 1$ such that the non-constant sequence $\left\{a_{n} / b_{n}\right\}_{n=0}^{\infty}$ is increasing (decreasing) for $0 \leq n \leq n_{0}$ and decreasing (increasing) for $n \geq n_{0}$, then there exists $x_{0} \in(0, r)$ such that the function $f(x) / g(x)$ is strictly increasing (decreasing) on $\left(0, x_{0}\right)$ and decreasing (increasing) on $\left(x_{0}, r\right)$.

Lemma 2.3 The function

$$
f(t)=\frac{\frac{2}{3} \log [\sec (t)]+\frac{t}{\tan (t)}-1}{\log \left[\frac{\sec ^{2}(t)+5}{6}\right]-\frac{\log [\sec (t)]}{3}}
$$


is strictly decreasing from $(0, \pi / 4)$ onto $(3 \pi+4 \log 2-12) /[2(6 \log 7-7 \log 2-6 \log 3)]$, $12 / 25)$.

Proof Let

$$
\begin{aligned}
& f_{1}(t)=\frac{2}{3} \log [\sec (t)]+\frac{t}{\tan (t)}-1, \\
& f_{2}(t)=\log \left[\frac{\sec ^{2}(t)+5}{6}\right]-\frac{\log [\sec (t)]}{3}, \\
& f_{3}(t)=2 \tan (t)+\sin (t) \cos (t)-3 t, \quad f_{4}(t)=\frac{5 \sin ^{5}(t)}{\cos (t)\left[1+5 \cos ^{2}(t)\right]} .
\end{aligned}
$$

Then it is not difficult to verify that

$$
\begin{aligned}
& f_{1}\left(0^{+}\right)=f_{2}\left(0^{+}\right)=f_{3}\left(0^{+}\right)=f_{4}\left(0^{+}\right)=0, \\
& f(t)=\frac{f_{1}(t)}{f_{2}(t)}, \quad \frac{f_{1}^{\prime}(t)}{f_{2}^{\prime}(t)}=\frac{f_{3}(t)}{f_{4}(t)}, \\
& \frac{f_{3}^{\prime}(t)}{f_{4}^{\prime}(t)}=\frac{2\left[1+5 \cos ^{2}(t)\right]^{2}}{5\left[10 \cos ^{4}(t)+19 \cos ^{2}(t)+1\right]}, \\
& {\left[\frac{f_{3}^{\prime}(t)}{f_{4}^{\prime}(t)}\right]^{\prime}=-\frac{2 \sin (2 t)\left[1+5 \cos ^{2}(t)\right]\left[75 \cos ^{2}(t)-9\right]}{5\left[10 \cos ^{4}(t)+19 \cos ^{2}(t)+1\right]^{2}}<0}
\end{aligned}
$$

for $t \in(0, \pi / 4)$.

Therefore, the function $f(t)$ is strictly decreasing on $(0, \pi / 4)$ follows easily from Lemma 2.1, (2.2), (2.3), and (2.5).

It follows from (2.1)-(2.4) that

$$
f\left(0^{+}\right)=\lim _{t \rightarrow 0^{+}} \frac{f_{3}^{\prime}(t)}{f_{4}^{\prime}(t)}=\frac{12}{25}
$$

and

$$
f\left(\frac{\pi}{4}\right)=\lim _{t \rightarrow \pi / 4} \frac{\frac{2}{3} \log [\sec (t)]+\frac{t}{\tan (t)}-1}{\log \left[\frac{\sec ^{2}(t)+5}{6}\right]-\frac{\log [\sec (t)]}{3}}=\frac{3 \pi+4 \log 2-12}{2(6 \log 7-7 \log 2-6 \log 3)}=0.4258 \ldots
$$

Lemma 2.4 The function

$$
g(t)=\frac{t \operatorname{coth}(t)-\frac{2 \log [\cosh (t)]}{3}-1}{\log \left[\frac{\cosh ^{2}(t)+2}{3}\right]-\frac{2 \log [\cosh (t)]}{3}}
$$

is strictly decreasing from $(0, \log (1+\sqrt{2}))$ onto $([3 \sqrt{2} \log (1+\sqrt{2})-\log 2-3] /(5 \log 2-$ $3 \log 3), 3 / 10)$.

Proof Let

$$
g_{1}(t)=t \operatorname{coth}(t)-\frac{2 \log [\cosh (t)]}{3}-1,
$$




$$
\begin{aligned}
& g_{2}(t)=\log \left[\frac{\cosh ^{2}(t)+2}{3}\right]-\frac{2 \log [\cosh (t)]}{3}, \\
& g_{3}(t)=\left[3 \sinh (t)+\sinh ^{3}(t)-3 t \cosh (t)\right]\left[\cosh ^{2}(t)+2\right], \\
& g_{4}(t)=4 \sinh ^{5}(t) .
\end{aligned}
$$

Then we clearly see that

$$
\begin{aligned}
& g_{1}\left(0^{+}\right)=g_{2}\left(0^{+}\right)=g_{3}\left(0^{+}\right)=g_{4}\left(0^{+}\right)=0, \\
& g(t)=\frac{g_{1}(t)}{g_{2}(t)}, \quad \frac{g_{1}^{\prime}(t)}{g_{2}^{\prime}(t)}=\frac{g_{3}(t)}{g_{4}(t)} .
\end{aligned}
$$

Elaborate computations lead to

$$
\begin{aligned}
\frac{g_{3}^{\prime}(t)}{g_{4}^{\prime}(t)} & =\frac{5 \sinh (4 t)+50 \sinh (2 t)-84 t-36 t \cosh (2 t)}{20[\sinh (4 t)-2 \sinh (2 t)]} \\
& =\frac{5 \sum_{n=0}^{\infty} \frac{(4 t)^{2 n+1}}{(2 n+1) !}+50 \sum_{n=0}^{\infty} \frac{(2 t)^{2 n+1}}{(2 n+1) !}-84 t-36 t \sum_{n=0}^{\infty} \frac{(2 t)^{2 n}}{(2 n) !}}{20 \sum_{n=0}^{\infty} \frac{(4 t)^{2 n+1}}{(2 n+1) !}-40 \sum_{n=0}^{\infty} \frac{(2 t)^{2 n+1}}{(2 n+1) !}}=: \frac{\sum_{n=0}^{\infty} a_{n}}{\sum_{n=0}^{\infty} b_{n}},
\end{aligned}
$$

where

$$
a_{n}=\frac{\left(5 \times 2^{2 n+1}-9 n-1\right) 2^{2 n+5}}{(2 n+3) !}, \quad b_{n}=\frac{10\left(2^{2 n+2}-1\right) 2^{2 n+5}}{(2 n+3) !} .
$$

From (2.10) we clearly see that

$$
\begin{aligned}
& \frac{a_{1}}{b_{1}}-\frac{a_{0}}{b_{0}}=-\frac{1}{10}<0, \\
& \frac{a_{n+1}}{b_{n+1}}-\frac{a_{n}}{b_{n}}=\frac{9\left[3(2 n-1) 2^{2 n+1}+1\right]}{10\left(2^{2 n+2}-1\right)\left(2^{2 n+4}-1\right)}>0
\end{aligned}
$$

for all $n \geq 1$, and

$$
b_{n}>0
$$

for all $n \geq 0$.

It follows from Lemma 2.2 and (2.9)-(2.13) that there exists $t_{0} \in(0, \infty)$ such that the function $g_{3}^{\prime}(t) / g_{4}^{\prime}(t)$ is strictly decreasing on $\left(0, t_{0}\right)$ and strictly increasing on $\left(t_{0}, \infty\right)$.

Note that

$$
\begin{aligned}
{\left[\frac{g_{3}^{\prime}(t)}{g_{4}^{\prime}(t)}\right]^{\prime}=} & \frac{4 \cosh (4 t)+16 \cosh (2 t)-18 t \sinh (2 t)-21}{5[\sinh (4 t)-2 \sinh (2 t)]} \\
& -\frac{[5 \sinh (4 t)+50 \sinh (2 t)-84 t-36 t \cosh (2 t)][\cosh (4 t)-\cosh (2 t)]}{5[\sinh (4 t)-2 \sinh (2 t)]^{2}} \\
{\left[\frac{g_{3}^{\prime}(t)}{g_{4}^{\prime}(t)}\right]_{t=\log (1+\sqrt{2})}^{\prime} } & =\frac{(13,464 \sqrt{2}+19,041) \log (1+\sqrt{2})-12,117 \sqrt{2}-17,136}{5770+4080 \sqrt{2}} \\
& =-0.0613 \ldots<0
\end{aligned}
$$


From (2.14) and piecewise monotonicity of the function $g_{3}^{\prime}(t) / g_{4}^{\prime}(t)$, we clearly see that $t_{0}>\log (1+\sqrt{2})$ and the function $g_{3}^{\prime}(t) / g_{4}^{\prime}(t)$ is strictly decreasing on $(0, \log (1+\sqrt{2}))$. Then Lemma 2.1 together with (2.7) and (2.8) leads to the conclusion that $g(t)$ is strictly decreasing on $(0, \log (1+\sqrt{2}))$.

It follows from (2.6)-(2.10) that

$$
g\left(0^{+}\right)=\frac{a_{0}}{b_{0}}=\frac{3}{10}, \quad g(\log (1+\sqrt{2}))=\frac{3 \sqrt{2} \log (1+\sqrt{2})-\log 2-3}{5 \log 2-3 \log 3}=0.2719 \ldots
$$

\section{Main results}

Theorem 3.1 The double inequality

$$
\begin{aligned}
{\left[\frac{1}{6}\right.} & \left.C(x, y)+\frac{5}{6} A(x, y)\right]^{\alpha_{1}}\left[C^{1 / 6}(x, y) A^{5 / 6}(x, y)\right]^{1-\alpha_{1}} \\
& <R_{A Q}(x, y) \\
& <\left[\frac{1}{6} C(x, y)+\frac{5}{6} A(x, y)\right]^{\beta_{1}}\left[C^{1 / 6}(x, y) A^{5 / 6}(x, y)\right]^{1-\beta_{1}}
\end{aligned}
$$

holds for all $x, y>0$ with $x \neq y$ if and only if $\alpha_{1} \leq(3 \pi+4 \log 2-12) /[2(6 \log 7-7 \log 2-$ $6 \log 3)]=0.4258 \ldots$ and $\beta_{1} \geq 12 / 25$.

Proof Since $A(x, y), R_{A Q}(x, y)$, and $C(x, y)$ are symmetric and homogenous of degree one, without loss of generality, we assume that $x>y>0$. Let $v=(x-y) /(x+y) \in(0,1)$ and $t=\arctan (v) \in(0, \pi / 4)$. Then (1.1)-(1.3) lead to

$$
\begin{aligned}
\frac{\log \left[R_{A Q}(x, y)\right]-\log \left[C^{1 / 6}(x, y) A^{5 / 6}(x, y)\right]}{\log [C(x, y) / 6+5 A(x, y) / 6]-\log \left[C^{1 / 6}(x, y) A^{5 / 6}(x, y)\right]} \\
=\frac{\log \left(\sqrt{1+v^{2}}\right)+\arctan (v) / v-1-\log \left(\sqrt[6]{1+v^{2}}\right)}{\log \left[\left(1+v^{2}\right) / 6+5 / 6\right]-\log \left(\sqrt[6]{1+v^{2}}\right)} \\
=\frac{\frac{2}{3} \log [\sec (t)]+\frac{t}{\tan (t)}-1}{\log \left[\frac{\sec ^{2}(t)+5}{6}\right]-\frac{\log [\sec (t)]}{3}} .
\end{aligned}
$$

Therefore, Theorem 3.1 follows easily from Lemma 2.3 and (3.1).

\section{Theorem 3.2 The two-sided inequalities}

$$
\begin{aligned}
{[} & \left.\frac{1}{3} C(x, y)+\frac{2}{3} A(x, y)\right]^{\alpha_{2}}\left[C^{1 / 3}(x, y) A^{2 / 3}(x, y)\right]^{1-\alpha_{2}} \\
& <R_{Q A}(x, y) \\
& <\left[\frac{1}{3} C(x, y)+\frac{2}{3} A(x, y)\right]^{\beta_{2}}\left[C^{1 / 3}(x, y) A^{2 / 3}(x, y)\right]^{1-\beta_{2}}
\end{aligned}
$$

are valid for all $x, y>0$ with $x \neq y$ if and only if $\alpha_{2} \leq[3 \sqrt{2} \log (1+\sqrt{2})-\log 2-3] /(5 \log 2-$ $3 \log 3)=0.2719 \ldots$ and $\beta_{2} \geq 3 / 10$. 
Proof Since $A(x, y), R_{Q A}(x, y)$, and $C(x, y)$ are symmetric and homogenous of degree one, without loss generality, we assume that $x>y>0$. Let $v=(x-y) /(x+y) \in(0,1)$ and $t=$ $\sinh ^{-1}(v) \in(0, \log (1+\sqrt{2})$. Then from (1.1), (1.3), and (1.4) we clearly see that

$$
\begin{aligned}
& \frac{\log \left[R_{Q A}(x, y)\right]-\log \left[C^{1 / 3}(x, y) A^{2 / 3}(x, y)\right]}{\log [C(x, y) / 3+2 A(x, y) / 3]-\log \left[C^{1 / 3}(x, y) A^{2 / 3}(x, y)\right]} \\
& =\frac{\left[\sqrt{1+v^{2}} \sinh ^{-1}(v)\right] / v-1-\log \left(\sqrt[3]{1+v^{2}}\right)}{\log \left[\left(1+v^{2}\right) / 3+2 / 3\right]-\log \left(\sqrt[3]{1+v^{2}}\right)} \\
& =\frac{t \operatorname{coth}(t)-\frac{2 \log [\cosh (t)]}{3}-1}{\log \left[\frac{\cosh ^{2}(t)+2}{3}\right]-\frac{2 \log [\cosh (t)]}{3}} .
\end{aligned}
$$

Therefore, Theorem 3.2 follows easily from Lemma 2.4 and (3.2).

From (1.3), (1.4), and Theorems 3.1 and 3.2 we get Corollary 3.3 immediately.

\section{Corollary 3.3 Let}

$$
\begin{aligned}
\lambda(\alpha ; a, b)= & 6 \alpha \log [C(a, b)+5 A(a, b)] \\
& +(1-\alpha)[\log C(a, b)+5 \log A(a, b)]-6 \log Q(a, b)+6(1-\alpha \log 6), \\
\mu(\alpha ; a, b)= & 3 \alpha \log [C(a, b)+2 A(a, b)] \\
& +(1-\alpha) \log C(a, b)-(1+2 \alpha) \log A(a, b)+3(1-\alpha \log 3) .
\end{aligned}
$$

Then the double inequalities

$$
\begin{aligned}
\frac{6 A(a, b)}{\lambda\left(\beta_{1} ; a, b\right)}<T(a, b)<\frac{6 A(a, b)}{\lambda\left(\alpha_{1} ; a, b\right)}, \\
\frac{3 Q(a, b)}{\mu\left(\beta_{2} ; a, b\right)}<N S(a, b)<\frac{3 Q(a, b)}{\mu\left(\alpha_{2} ; a, b\right)}
\end{aligned}
$$

hold for all $a, b>0$ with $a \neq b$ if and only if $\alpha_{1} \leq(3 \pi+4 \log 2-12) /[2(6 \log 7-7 \log 2-$ $6 \log 3)]=0.4258 \ldots, \beta_{1} \geq 12 / 25, \alpha_{2} \leq[3 \sqrt{2} \log (1+\sqrt{2})-\log 2-3] /(5 \log 2-3 \log 3)=$ $0.2719 \ldots$, and $\beta_{2} \geq 3 / 10$.

\section{Results and discussion}

In the article, we present the best possible parameters $\alpha_{1}, \beta_{1}, \alpha_{2}$, and $\beta_{2}$ such that the double inequalities

$$
\begin{aligned}
& {\left[\frac{1}{6} C(x, y)+\frac{5}{6} A(x, y)\right]^{\alpha_{1}}\left[C^{1 / 6}(x, y) A^{5 / 6}(x, y)\right]^{1-\alpha_{1}}} \\
& <R_{A Q}(x, y) \\
& \quad<\left[\frac{1}{6} C(x, y)+\frac{5}{6} A(x, y)\right]^{\beta_{1}}\left[C^{1 / 6}(x, y) A^{5 / 6}(x, y)\right]^{1-\beta_{1}}, \\
& {\left[\frac{1}{3} C(x, y)+\frac{2}{3} A(x, y)\right]^{\alpha_{2}}\left[C^{1 / 3}(x, y) A^{2 / 3}(x, y)\right]^{1-\alpha_{2}}}
\end{aligned}
$$




$$
\begin{aligned}
& <R_{Q A}(x, y) \\
& <\left[\frac{1}{3} C(x, y)+\frac{2}{3} A(x, y)\right]^{\beta_{2}}\left[C^{1 / 3}(x, y) A^{2 / 3}(x, y)\right]^{1-\beta_{2}}
\end{aligned}
$$

hold for all $x, y>0$ with $x \neq y$. Our results are the improvements of the inequalities given by (1.5) and (1.6).

\section{Conclusion}

We present sharp upper and lower bounds for the Sándor-Yang means $R_{A Q}$ and $R_{Q A}$ in terms of the arithmetic and contraharmonic means and provide new bounds for the Seiffert mean $T$ and Neuman-Sándor mean NS. Our approach may have further applications in the theory of bivariate means and special functions.

\section{Funding}

The research was supported by the Natural Science Foundation of China under Grants 61673169, 11301127, 11701176, 11626101, and 11601485, the Natural Science Foundation of Zhejiang Broadcast and TV University under Grant XKT-17Z04, and the Natural Science Foundation of Huzhou City under Grant 2018 YZ07.

\section{Competing interests}

The authors declare that they have no competing interests.

\section{Authors' contributions}

All authors contributed equally to the writing of this paper. All authors read and approved the final manuscript.

\section{Author details}

'School of Continuing Education, Huzhou Vocational \& Technical College, Huzhou, China. ${ }^{2}$ School of Economics and Management, Wenzhou Broadcast and TV University, Wenzhou, China. ${ }^{3}$ Department of Mathematics, Huzhou University, Huzhou, China.

\section{Publisher's Note}

Springer Nature remains neutral with regard to jurisdictional claims in published maps and institutional affiliations.

Received: 31 October 2018 Accepted: 15 March 2019 Published online: 21 March 2019

\section{References}

1. Neuman, E.: Sharp inequalities involving Neuman-Sándor and logarithmic means. J. Math. Inequal. 7(3), 413-419 (2013)

2. Chu, Y.-M., Zong, C., Wang, G.-D.: Optimal convex combination bounds of Seiffert and geometric means for arithmetic mean. J. Math. Inequal. 5(3), 429-434 (2011)

3. Chu, Y.-M., Hou, S.-W.: Sharp bounds for Seiffert mean in terms of contraharmonic mean. Abstr. Appl. Anal. 2012, Article ID 425175 (2012)

4. Chu, Y.-M., Wang, M.-K., Wang, G.-D.: The optimal generalized logarithmic mean bounds for Seiffert mean. Acta Math. Sci. 32B(4), 1619-1626 (2012)

5. Chu, Y.-M., Wang, M.-K., Wang, Z.-K.: Best possible inequalities among harmonic, geometric, logarithmic and Seiffert means. Math. Inequal. Appl. 15(2), 415-422 (2012)

6. Lin, T.P.: The power and the logarithmic mean. Am. Math. Mon. 81, 879-883 (1974)

7. Alzer, H., Qiu, S.-L.: Inequalities for means in two variables. Arch. Math. 80(2), 201-215 (2003)

8. Yang, Z.-H., Qian, W.-M., Chu, Y.-M., Zhang, W.: Monotonicity rule for the quotient of two functions and its application. J. Inequal. Appl. 2017, Article ID 106 (2017)

9. Chu, Y.-M., Xia, W.-F.: Two optimal double inequalities between power mean and logarithmic mean. Comput. Math. Appl. 60(1), 83-89(2010)

10. Chu, Y.-M., Qiu, Y.-F., Wang, M.-K.: Sharp power mean bounds for combination of Seiffert and geometric means. Abstr. Appl. Anal. 2010, Article ID 108920 (2010)

11. Chu, Y.-M., Wang, S.-S., Zong, C.: Optimal lower power mean bound for the convex combination of harmonic and logarithmic means. Abstr. Appl. Anal. 2011, Article ID 520648 (2011)

12. Wang, G.-D., Zhang, X.-H., Chu, Y.-M.: A power mean inequality for the Grötzsch ring function. Math. Inequal. Appl. 14(4), 833-837 (2011)

13. Wang, G.-D., Zhang, X.-H., Chu, Y.-M.: A power mean inequality involving the complete elliptic integrals. Rocky Mt. J. Math. 44(5), 1661-1667 (2014)

14. Neuman, E., Sándor, J.: On the Schwab-Borchardt mean. Math. Pannon. 14(2), 253-266 (2003)

15. Neuman, E., Sándor, J.: On the Schwab-Borchardt mean II. Math. Pannon. 17(1), 49-59 (2006)

16. Yang, Z.-H.: Three families of two-parameter means constructed by trigonometric functions. J. Inequal. Appl. 2013, Article ID 541 (2013) 
17. Zhao, T.-H., Wang, M.-K., Chu, Y.-M.: Quadratic transformation inequalities for Gaussian hypergeometric function. J. Inequal. Appl. 2018, Article ID 251 (2018)

18. Chu, Y.-M., Wang, M.-K., Qiu, S.-L.: Optimal combinations bounds of root-square and arithmetic means for Toader mean. Proc. Indian Acad. Sci. Math. Sci. 122(1), 41-51 (2012)

19. Chu, Y.-M., Wang, M.-K.: Optimal Lehmer mean bounds for the Toader mean. Results Math. 61(3-4), 223-229 (2012)

20. Qian, W.-M., Chu, Y.-M.: Sharp bounds for a special quasi-arithmetic mean in terms of arithmetic and geometric means with two parameters. J. Inequal. Appl. 2017, Article ID 274 (2017)

21. Yang, Z.-H., Qian, W.-M., Chu, Y.-M.: On rational bounds for the gamma function. J. Inequal. Appl. 2017, Article ID 210 (2017)

22. Adil Khan, M., Chu, Y.-M., Khan, T.U., Khan, J.: Some new inequalities of Hermite-Hadamard type for s-convex functions with applications. Open Math. 15, 1414-1430 (2017)

23. Yang, Z.-H., Qian, W.-M., Chu, Y.-M.: Monotonicity properties and bounds involving the complete elliptic integrals of the first kind. Math. Inequal. Appl. 21(4), 1185-1199 (2018)

24. Yang, Z.-H., Chu, Y.-M., Zhang, W.: High accuracy asymptotic bounds for the complete elliptic integral of the second kind. Appl. Math. Comput. 348, 552-564 (2019)

25. Khurshid, Y., Adil Khan, M., Chu, Y.-M., Khan, Z.A., Liu, L.-S.: Hermite-Hadamard-Fejér inequalities for conformable fractional integrals via preinvex functions. J. Funct. Spaces 2019, Article ID 3146210 (2019)

26. Khurshid, Y., Adil Khan, M., Chu, Y.-M.: Conformable integral inequalities of the Hermite-Hadamard type in terms of GG- and GA-convexities. J. Funct. Spaces 2019, Article ID 6926107 (2019)

27. Wang, J.-L., Qian, W.-M., He, Z.-Y., Chu, Y.-M.: On approximating the Toader mean by other bivariate means. J. Funct. Spaces 2019, Article ID 6082413 (2019)

28. Qiu, S.-L., Ma, X.-Y., Chu, Y.-M.: Sharp Landen transformation inequalities for hypergeometric functions, with applications. J. Math. Anal. Appl. 474(2), 1306-1337 (2019)

29. Zhao, T.-H., Qian, W.-M., Song, Y.-Q.: Optimal bounds for two Sándor-type means in terms of power means. J. Inequal. Appl. 2016, Article ID 64 (2016)

30. $\mathrm{Xu}, \mathrm{H} . \mathrm{Z}$-: Sharp bounds for Sándor-Yang means in terms of some bivariate means. J. East China Norm. Univ. Natur. Sci. Ed. 2017(4), 41-51 (2017)

31. Xu, H.-Z., Chu, Y.-M., Qian, W.-M.: Sharp bounds for the Sándor-Yang means in terms of arithmetic and contra-harmonic means. J. Inequal. Appl. 2018, Article ID 127 (2018)

32. Anderson, G.D., Vamanamurthy, M.K., Vuorinen, M.: Conformal Invariants, Inequalities, and Quasiconformal Maps. Wiley, New York (1997)

33. Simić, S., Vuorinen, M.: Landen inequalities for zero-balanced hypergeometric functions. Abstr. Appl. Anal. 2012, Article ID 932061 (2012)

\section{Submit your manuscript to a SpringerOpen ${ }^{\circ}$ journal and benefit from:}

- Convenient online submission

- Rigorous peer review

- Open access: articles freely available online

- High visibility within the field

- Retaining the copyright to your article

Submit your next manuscript at $\gg$ springeropen.com 